\title{
Systematic design of output filters for audio class-D amplifiers via Simplified Real Frequency Technique
}

\author{
E. Hintzen, T. Vennemann, and W. Mathis \\ Institute for Theoretical Electrical Engineering, Leibniz Universität Hannover, Hannover, Germany \\ Correspondence to: T. Vennemann (vennemann@tet.uni-hannover.de)
}

Received: 26 January 2014 - Revised: 5 May 2014 - Accepted: 9 May 2014 - Published: 10 November 2014

\begin{abstract}
In this paper a new filter design concept is proposed and implemented which takes into account the complex loudspeaker impedance. By means of techniques of broadband matching, that has been successfully applied in radio technology, we are able to optimize the reconstruction filter to achieve an overall linear frequency response. Here, a passive filter network is inserted between source and load that matches the complex load impedance to the complex source impedance within a desired frequency range. The design and calculation of the filter is usually done using numerical approximation methods which are known as Real Frequency Techniques (RFT). A first approach to systematic design of reconstruction filters for class-D amplifiers is proposed, using the Simplified Real Frequency Technique (SRFT). Some fundamental considerations are introduced as well as the benefits and challenges of impedance matching between class-D amplifiers and loudspeakers. Current simulation data using MATLAB is presented and supports some first conclusions.
\end{abstract}

\section{Introduction and motivation}

The development of audio technology in recent years is primarily characterized by more efficient and compact power components and systems. Due to the rapid growth and ongoing development of mobile devices, class-D amplifiers have become prevalent in modern applications.

The concept of class-D amplifiers can handle only switching signals, so the circuit complexity is much higher compared to linear power amplifier concepts to guarantee an overall linear signal transmission. The audio signal has to be initially encoded by a modulator (e.g. pulse width modulation). Then, the signal can be amplified by the switching stage and finally it has to be decoded by a demodulator (reconstruction filter). An alternative encoding method is called ZePoC (Zero Position Coding). ZePoC was initially used and implemented for audio coding by the Institute for Theoretical Electrical Engineering in Hannover. Much research was done over years resulting in a number of publications. A good overview could found in the white paper from Oliva et al. (2005).

The reconstruction filter is usually realized with conventional filter design techniques by defining pass and stop band and setting desired ripple and tolerance. Then, the transfer function is calculated with the help of well-known approximation procedures like Butterworth, Tschebyscheff or Cauer (Quek, 2006). A further work regarding the optimization of class-D output filter has been published by Doy (2002). In practice, a purely resistive load is often used to model the loudspeaker impedance at the output of the filter, thus neglecting complete frequency dependencies of real loudspeakers. In consequence of committing these effects, the frequency response of the filter will no longer be linear in the audio band and will distort the signal. This contribution deals with a new method of class-D output filter design which allows the designer to take into account the given impedance for an optimum overall frequency response. Therefore, some basic preliminaries and specifications have to be made first.

\section{Specifications for class-D filter design}

An existing class-D amplifier platform which has been introduced and successfully developed by the Institute for Theoretical Electrical Engineering in Hannover is used for a first filter design. Based on the sample rate frequency of $f_{\mathrm{s}}=$ $48 \mathrm{kHz}$ for pulse code modulation systems (PCM) like CD or DVD players, and taking account of the Nyquist-Shannon 


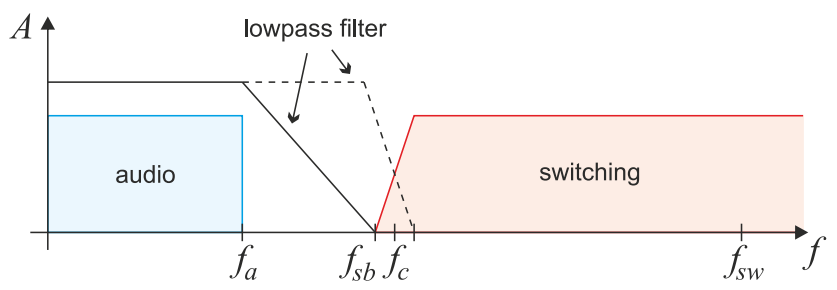

Figure 1. Frequency spectrum with audio and switching band.

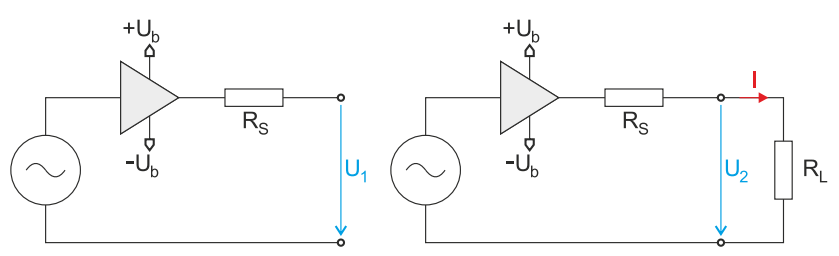

Figure 2. Output impedance measurement.

sampling theorem, the maximum signal frequency is limited to $24 \mathrm{kHz}$. However, the human ear is only capable of detecting frequencies up to $20 \mathrm{kHz}$, thus the audio frequency band can be defined from $20 \mathrm{~Hz}$ to $f_{\mathrm{a}}=20 \mathrm{kHz}$. The frequency response of the overall circuit, which consists of class-D output impedance, reconstruction filter and loudspeaker impedance, has to be completely flat within this entire wave band. Further on, the carrier frequency of the single-sideband $\mathrm{ZePoC}$ modulation is set to $f_{\mathrm{c}}=50 \mathrm{kHz}$ and then the switching frequency of the class-D amplifier is given by $f_{\mathrm{sw}}=2 f_{\mathrm{c}}=100 \mathrm{kHz}$. The resulting frequency spectrum is shown in Fig. 1.

Since the radio frequency energy initially rises slowly, the transition band of the reconstruction filter can be arranged symmetrically to the carrier frequency. On the other hand, the frequency band between $f_{\mathrm{a}}$ and $f_{\mathrm{sb}}$ does not contain any information, so that the transition band can be moved towards the audio band. In both cases, a sufficiently high attenuation should be reached at $f_{\mathrm{sb}}$ to prevent distortion. Before introducing the SRFT algorithm, the impedances on the input and output side of the filter are described.

\subsection{Class-D output impedance}

The output impedance of a class-D switching amplifier is among other things dependent on the used power supply, but essentially it is determined by the resistance of the power transistors. Since the output voltage of the switching amplifier is a pulse width modulated signal with a fixed switching frequency, it is assumed that the output impedance of the amplifier can be accepted as a constant within the considered frequency band. Typically, the output resistance of a class-D switching amplifier is in the range of only a few milliohms.

A precise mathematical description or even the calculation of the output resistance of a switching amplifier is only possible with high effort. Therefore, the resistance is determined

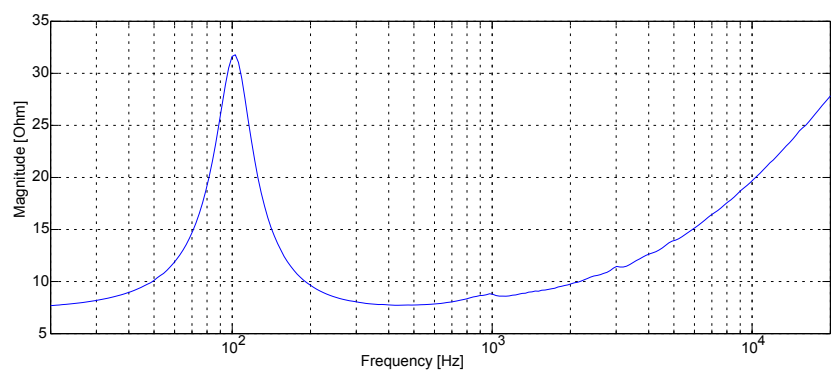

Figure 3. Measured impedance response of $8 \Omega$ fullrange speaker.

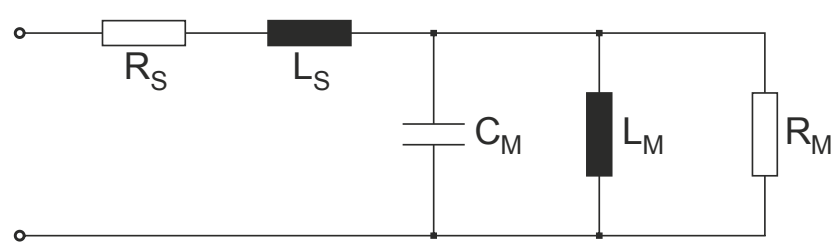

Figure 4. Electrical equivalent circuit of dynamic loudspeaker.

by a simple measurement procedure which is presented in Metzler (1993). In a first measurement, the amplifier is operated at no-load and a sine wave generator with fixed frequency is used as a signal source. The generator causes a defined voltage at the amplifiers output which is measured. In a second step, the amplifier is operated with a definded load and the voltage drop is measured. Then, knowing differential voltage $U_{1}-U_{2}$ and current through load resistor $I=\frac{U_{2}}{R_{\mathrm{L}}}$, the output resistance is given by $R_{\mathrm{S}}=R_{\mathrm{L}} \cdot\left(\frac{U_{1}}{U_{2}}-1\right)$. The measurement setup is shown in Fig. 2. By applying this measurement method, the output resistance of the class-D amplifier can be computed approximately to be $R_{0}=100 \mathrm{~m} \Omega$.

\subsection{Loudspeaker impedance}

The SRFT is a numerical approximation procedure and can handle real frequency measurement and simulation data. This also means that the filter design has to be done specifically for one type of loudspeaker and the choice of a speaker has to be made during the preliminary stages. For the first design a 6.5" fullrange speaker with a nominal impedance of $8 \Omega$ is used. Measuring the loudspeaker impedance is done by using a software which has to be extended by a simple measurement setup. The measured impedance response of the speaker is shown in Fig. 3.

The impedance starts with DC resistance of the voice coil at about $8 \Omega$. The magnitude increases with the selfresonance of the speaker cone around $100 \mathrm{~Hz}$ to more than $30 \Omega$, followed by a linear frequency range at around $4 \mathrm{kHz}$. Here, the impedance is purely resistive. Finally, the inductance of the voice coil dominates the loudspeaker impedance at around $10 \mathrm{kHz}$ and the magnitude increases steadily. In addition, for simulation purposes and to verify later results, an electrical equivalent circuit of the loudspeaker is needed. A 


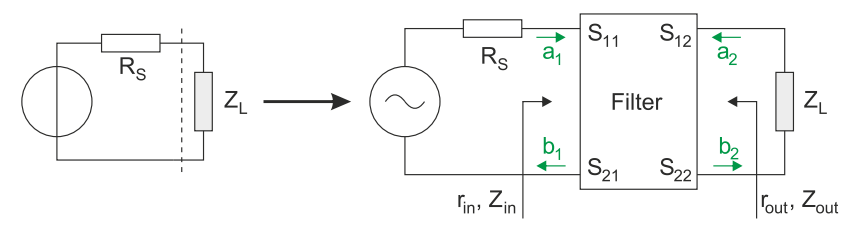

Figure 5. Single matching problem regarded as a two-port.

very simple equivalent circuit of a dynamic speaker is shown in Fig. 4. It neglects skin and hysteresis effects which primarily contribute to the increase in impedance at high frequencies. Measured data or the technical data sheet of the loudspeaker can be used to calculate the equivalent circuit elements.

\section{Simplified Real Frequency Technique}

The Simplified Real Frequency Technique (SRFT) is an alternative approach to the Real Frequency Technique (Carlin, 1977) and was first introduced in the work of Yarman (1982). This technique was developed for complex matching problems between generator and load in radio frequency and describes a given network regarded as a two-port as shown in Fig. 5.

In general, a two-port is described by its two-port parameters. The SRFT uses scattering parameters (S-parameters) to describe the electrical behavior of a lossless network by

$$
\left(\begin{array}{l}
b_{1} \\
b_{2}
\end{array}\right)=\left(\begin{array}{ll}
S_{11}(p) & S_{12}(p) \\
S_{21}(p) & S_{22}(p)
\end{array}\right)\left(\begin{array}{l}
a_{1} \\
a_{2}
\end{array}\right)
$$

Using Darlington-Cauer-Piloty's theorem (Darlington, 1939), the network can be described as a ladder network with multiple two-ports. The resulting network is shown in Fig. 6. The SRFT algorithm uses the transducer power gain (TPG) of a network for optimization which is definded by

$T=\frac{P_{\mathrm{L}}}{P_{\mathrm{S}, \max }}$,

where $P_{\mathrm{L}}$ is power to the load and $P_{\mathrm{S}, \text { max }}$ is maximum available power from source. Now, the purpose of the SRFT is to achieve the best possible TPG over a desired frequency band. Further, TPG of the ladder network in Fig. 6 can be expressed as a function of its two-port parameters by

$T(\omega)=\left|G_{21}\right|^{2} \frac{\left|E_{21}\right|^{2}}{\left|1-E_{11} G_{22}\right|^{2}\left|1-\hat{E}_{22} L_{11}\right|^{2}}\left|L_{21}\right|^{2}$

with

$\hat{E}_{22}=E_{22}+\frac{E_{21}^{2} G_{22}}{1-E_{11} G_{22}}$

being the output reflection coefficient of the filter. The twoport parameters of source $G_{\mathrm{ij}}$ and load $L_{\mathrm{ij}}$ are known since

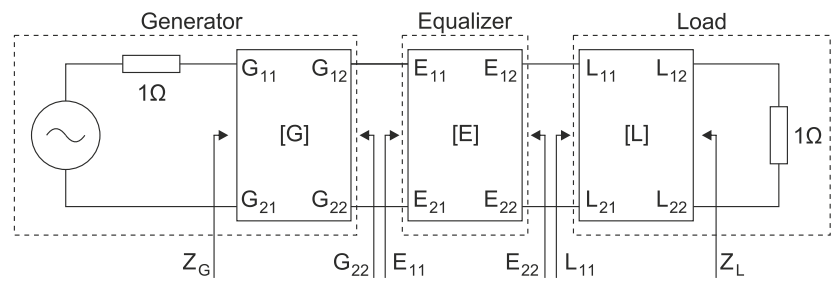

Figure 6. Equivalent network using Darlington's theorem.

they can be simply determined by measurement (Yarman, 2010).

The crux of SRFT is to generate the unknown parameters $E_{\mathrm{ij}}$ of the equalizer using Belevitch's theorem (Belevitch, 1968). The theorem states that an ideal reciprocal lossless two-port network can only be realized if the $\mathbf{S}$-matrix is given by

$\mathbf{S}(p)=\frac{1}{g(p)}\left(\begin{array}{cc}h(p) & f(p) \\ f(p) & -\frac{f(-p) h(-p)}{f(p)}\end{array}\right)$,

where $g(p)$ is a strict Hurwitz polynomial of degree $n$ (minimum number of inductors and capacitors) and $h(p)$ a real polynomial with $\operatorname{deg}(h) \leq \operatorname{deg}(g)$. The transmission zeros of the transfer function are represented in $f(p)$. Since the matching problem between amplifier and loudspeaker is considered to be a lowpass problem, we may choose all transmission zeros at infinity. This choice corresponds to setting $f(p)=1$.

The coefficients $h_{\mathrm{n}}$ of the polynomial $h(p)$ are unknown and chosen as initial values. In order to determine the coefficients $g_{\mathrm{n}}$ of the Hurwitz polynomial $g(p)$ as a function of the initial values, a numercial procedure is used. Once, the polynomial $g(p)$ is constructed, the S-parameters and TPG can be computed using Eqs. (3) and (4). For optimization, an error function is defined by

$$
\begin{aligned}
T_{\mathrm{E}}(\omega) & =T(\omega)-T_{0} \\
& =\left|G_{21}\right|^{2} \frac{\left|E_{21}\right|^{2}}{\left|1-E_{11} G_{22}\right|^{2}\left|1-\hat{E}_{22} L_{11}\right|^{2}}\left|L_{21}\right|^{2}-T_{0}
\end{aligned}
$$

with $T_{0}$ being the desired TPG. As a result of optimization, the coefficients $h_{\mathrm{n}}$ of $h(p)$ are known. Finally, the LC filter is constructed by synthesis of the driving-point impedance which is given by

$$
Z_{\text {in }}(p)=\frac{a(p)}{b(p)}=\frac{1+E_{22}}{1-E_{22}}=\frac{g(p)+h(p)}{g(p)-h(p)} .
$$

As a consequence of the choice of all transmission zeros at infinity, the input impedance $Z_{\text {in }}(p)$ may be realized by the well-known Cauer canonic realization using continued 


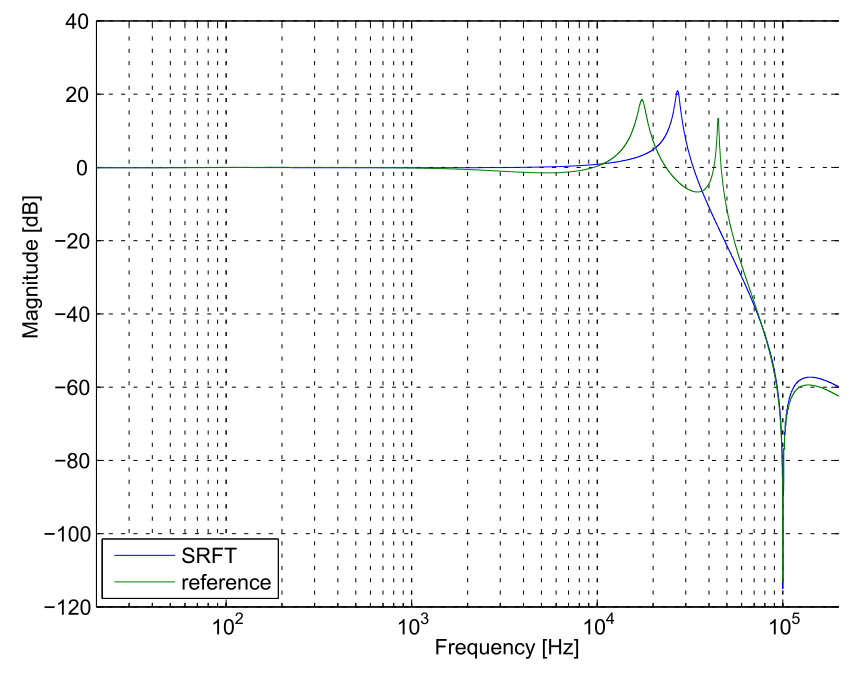

Figure 7. Frequency response of reference and computed SRFT filter.

fraction expansion about $p=\infty$ :

$$
Z_{\text {in }}(p)=Z_{1}(p)+\frac{1}{Y_{2}(p)+\frac{1}{Z_{3}(p)+\frac{1}{\cdots}}} .
$$

The terms $Z_{\mathrm{i}}(p)$ are purely imaginary impedances (inductors), whereas $Y_{\mathrm{i}}(p)$ are purely imaginary admittances (capacitors). Thus, a LC ladder network can be realized directly by use of this expression.

\section{Simulation using MATLAB}

In a series of simulations especially one type of filter has been found which is going be introduced briefly in the following. In Fig. 7 the computed frequency response of the SRFT filter is presented. A common Butterworth filter is used for reference. It can be seen that the response of the SRFT filter is maximum flat within the frequency band of $0 \mathrm{~Hz}$ to $10 \mathrm{kHz}$, whereas the response of the reference filter has some ripple at around $5 \mathrm{kHz}$. Further on, the resonance peak of the reference filter is at around $18 \mathrm{kHz}$ which means that the resonance is within the audio frequency band. The peak of the SRFT filter is shifted towards higher frequencies, thus the response is longer flat. In both cases, the stop band attenuation is sufficiently high. The frequency response can be optimized even further by inserting an additional transmission zero to the transfer function nearby the switching frequency. This is done by connecting a further capacitor $C_{\mathrm{P}}$ in parallel with $L_{2}$ as shown in Fig. 8. The computed filter element values are: $L_{1}=392.5 \mathrm{nH}, L_{2}=5.26 \mu \mathrm{H}, C_{1}=81.2 \mu \mathrm{F}, C_{2}=6.72 \mu \mathrm{F}$ and $C_{\mathrm{P}}=481.56 \mathrm{nF}$.

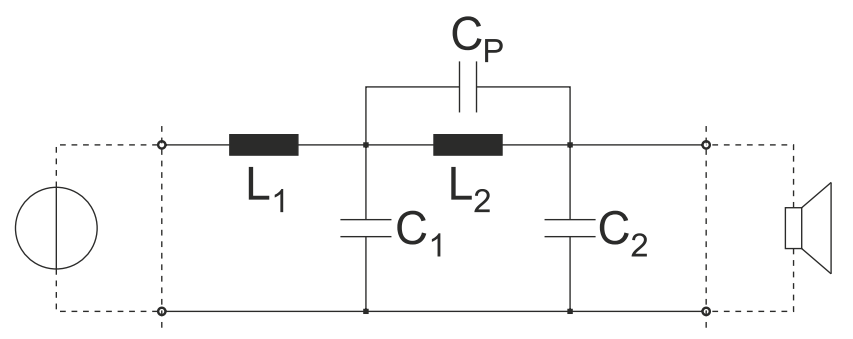

Figure 8. Realized LC ladder network of computed SRFT filter.

\section{Conclusions}

In this paper, the systematic design of output filters for audio class-D amplifiers via SRFT is presented. Therefore, a short introduction of specifications for filter design was given. After describing the class-D output impedance and the loudspeaker impedance, the SRFT algorithm was presented. Finally, some first simulation results were presented using MATLAB and the computed LC ladder network was illustrated. The simulations also revealed that the optimization criterion (TPG) is not chosen well, since there's usually no power adjustment in audio electronic but voltage adaption. Thus, the computed frequency responses are mostly independend of the desired TPG. Further work could deal with adaption of the optimization criterion.

Edited by: J. Götze

Reviewed by: two anonymous referees

\section{References}

Belevitch, V.: Classical Network Theory, Holden Day, San Francisco, 276-279 1968.

Carlin, H. J.: A New Approach to Gain-Bandwidth Problems, IEEE T. Circuit Theory, CAS-24, 4, April 1977.

Darlington, S.: Synthesis of Reactance 4-poles, J. Math. Phys. Camb., 18, 257-353, 1939.

Doy, T.: Class D Audio Amplifier Output Filter Optimization, Application Note, Maxim Integrated Products, Sunnyvale, 8 pp., 2002.

Metzler, B.: Audio Measurement Handbook, Audio Precision, Beaverton, 59-60, 1993.

Quek, Y. B.: Class-D LC Filter Design, Application Report, Texas Instruments, 20 pp., April 2006.

Oliva, A., Paolini, E., and Ang, S. S.: A New Audio File Format for Low-Cost, High Fidelity, Portable Digital Audio Amplifiers, 15 pp., Texas Instruments, White Paper, October 2005.

Yarman, B. S.: A Simplified Real Frequency Technique for Broadband Matching Complex Generator to Complex Loads, RCA Review, 43, 529-541, September 1982.

Yarman, B. S.: Design of Ultra Wideband Power Transfer Networks, Wiley Chichester, 616-619, 2010. 